\title{
The Computational Fluid Dynamics Study of Orientation Effects of Oar Blade
}

\author{
Alexandra Laurent, ${ }^{1}$ Annie Rouard, ${ }^{1}$ Vishveshwar R. Mantha, ${ }^{2,3}$ Daniel A. Marinho, ${ }^{3,4}$ \\ Antonio J. Silva, ${ }^{2}$ and Abel I. Rouboa ${ }^{2,5,6}$ \\ ${ }^{1}$ University of Savoie; ${ }^{2}$ University of Trás-os-Montes-e-Alto Douro; ${ }^{3}$ Research Centre in Sports, \\ Health and Human Development, Vila Real; ${ }^{4}$ University of Beira Interior; ${ }^{5}$ Research Centre and \\ Technologies of Agro-Environment and Biological Sciences, Vila Real; 6 University of Pennsylvania
}

\begin{abstract}
The distribution of pressure coefficient formed when the fluid contacts with the kayak oar blade is not been studied extensively. The CFD technique was employed to calculate pressure coefficient distribution on the front and rear faces of oar blade resulting from the numerical resolution equations of the flow around the oar blade in the steady flow conditions $(4 \mathrm{~m} / \mathrm{s})$ for three angular orientations of the oar $\left(45^{\circ}, 90^{\circ}, 135^{\circ}\right)$ with main flow. A three-dimensional (3D) geometric model of oar blade was modeled and the kappa-epsilon turbulence model was applied to compute the flow around the oar. The main results reported that, under steady state flow conditions, the drag coefficient $\left(C_{d}=2.01\right.$ for $\left.4 \mathrm{~m} / \mathrm{s}\right)$ at $90^{\circ}$ orientation has the similar evolution for the different oar blade orientation to the direction of the flow. This is valid when the orientation of the blade is perpendicular to the direction of the flow. Results indicated that the angle of oar strongly influenced the $\mathrm{C}_{\mathrm{d}}$ with maximum values for $90^{\circ}$ angle of the oar. Moreover, the distribution of the pressure is different for the internal and external edges depending upon oar angle. Finally, the difference of negative pressure coefficient $\mathrm{C}_{\mathrm{p}}$ in the rear side and the positive $\mathrm{C}_{\mathrm{p}}$ in the front side, contributes toward propulsive force. The results indicate that CFD can be considered an interesting new approach for pressure coefficient calculation on kayak oar blade. The CFD approach could be a useful tool to evaluate the effects of different blade designs on the oar forces and consequently on the boat propulsion contributing toward the design improvement in future oar models. The dependence of variation of pressure coefficient on the angular position of oar with respect to flow direction gives valuable dynamic information, which can be used during training for kayak competition.
\end{abstract}

Keywords: kayak oar blade, computational fluid dynamics, pressure coefficient, propulsion

Kayak rowing is a competitive sport, and the kayak speed is related to three components: (i) the strength and the coordination of the kayaker which determine the force

\footnotetext{
Alexandra Laurent and Annie Rouard are with the Sport Science Department, University of Savoie, Chambery, France. Vishveshwar R. Mantha is with the Engineering Department, University of Trás-os-Montes-e-Alto Douro, Vila Real, Portugal, and with the Research Centre in Sports, Health and Human Development (CIDESD), Vila Real, Portugal. Daniel A. Marinho is with the Research Centre in Sports, Health and Human Development (CIDESD), Vila Real, Portugal, and with the Department of Sport Sciences, University of Beira Interior, Covilhã, Portugal. Antonio J. Silva is with the Engineering Department and with the Department of Sport Sciences, Exercise and Health, University of Trás-os-Montes-e-Alto Douro, Vila Real, Portugal. Abel I. Rouboa (Corresponding Author) is with the Engineering Department, University of Trás-os-Montes-e-Alto Douro, Vila Real, Portugal; the Research Centre and Technologies of AgroEnvironment and Biological Sciences (CITAB), Vila Real, Portugal; and the Department of Mechanical Engineering and Applied Mechanics, University of Pennsylvania, Philadelphia, PA.
}

transmission from the kayaker to the blade and to the boat (Aitken \& Neal, 1992), (ii) the design of the boat which determines the drag (i.e., the force exerted by the water opposite to the boat displacement; Grare, 1985), (iii) the oar-blade system which transmits the force exerted by the kayaker (Mann \& Kearney, 1980; Ackland, 2003). Different studies focused on the optimization of the race, concluding to the relationship between boat velocity and cycle parameters (frequency or length) related to kayaker's power (Dolnik \& Krasnopievtsef, 1981). A few studies were concerned with the kinematics of the blades for various paddling techniques (Plagenhoef, 1979; Issurin, 1980; Issurin et al., 1983; Kendal \& Sanders, 1992). They concluded that the path of the blade must be parallel to the axis of the motion with a blade perpendicular to the boat during the propulsive phase. As the blade acts on the water, the blade did not remain fixed but presented slipping movement (Novakova, 1979; Plagenhoef, 1979; Mann \& Kearney, 1980; Issurin, 1980; Issurin et al., 1983; Kendal \& Sander, 1992; Di Puccio \& Mattei, 2008). It was concluded that the flat blade allowed for increasing the boat velocity more quickly at the beginning of the race, whereas the curved blade would allow a gain of boat pace 
during the race (Wargnier, 1990). Moreover, Kendal \& Sander (1992) observed that the velocity with a curved blade is related to greater forward attack and to a blade path close to the boat axis. Few studies concerned the force exerted by the blade, which appeared determinant of the boat velocity (Issurin, 1980; Issurin et al., 1983). A few studies are published on the hydrodynamic of the blade although the kayak factories developed different designs with variation of shape, curvature and width.

Kayak paddle can be compared with a swimmer's hand, which has been investigated from hydrodynamic approach (Marinho et al., 2010). The kayaker's force (or the swimmer's force) is transmitted to the blade (or the swimmer's hand), which propels the boat (or the body) through the water. The drag force is perpendicular to the direction of the movement of the hand or of the blade (Issurin, 1980; Issurin et al., 1983).

In swimming, based on kinematics or CFD, the drag force is strongly related to the orientation, the speed of the hand (Schleihauf, 1974; Berger et al., 1997; Rouboa et al. 2006), the size of limbs (Rushall et al., 1994; Berger et al., 1995; Gardano \& Dabnichki, 2005; Marinho et al., 2008; Minetti et al., 2009), and the position of the fingers (Schleihauf, 1974; Takagi et al., 2001; Minetti et al., 2009; Marinho et al., 2010). Moreover, the drag coefficient is constant for all flow speed and all sweep back angles (Bixler \& Riewald, 2002; Silva et al., 2005; Rouboa et al., 2006; Alves et al., 2007; Marinho et al., 2010). More recently, using 2D-CFD analysis, Gourgoulis observed that the paddle increased the efficiency of the propulsion and the length of the stroke cycle (Gourgoulis et al., 2008).

In regard to (i) the new blade development, (ii) the lack of study on the hydrodynamic characteristics of the blades and (iii) the previous results obtained in swimming, the main goal of the current study is to evaluate the influence of the blade angle on the flow, in particular focusing on the local pressure coefficient using the CFD approach. We hypothesized that (i) the local pressure coefficient is not similar on all the surface of the paddle, with different values between the front and rear faces, (ii) the drag coefficient changes with the angle of the blade $\left(45^{\circ}, 90^{\circ}, 135^{\circ}\right)$, with a maximal value for the blade perpendicular to the boat. These propositions are examined by 3D CFD approach as previous studies underlined the greater precision with 3D approach as compared with 2D approach (Rouboa et al., 2006; Zaidi et al., 2008). Results indicated that irrespective of the oar angle, the three $\mathrm{Cp}$ lines of the frontal side of the oar presented the similar shape but presented minor variation in $\mathrm{Cp}$ values along the reference line. Greater $\mathrm{Cp}$ was observed on the front face of the blade than on the rear one, irrespective the orientation of the blade was $\left(45^{\circ}, 90^{\circ}\right.$ and $\left.135^{\circ}\right)$. The pathlines colored with average flow velocity starting from frontal face of oar blade show recirculation zone at rear face of oar blade and average velocity around the kayak oar with rear side view, the pressure and pressure coefficient on the front side of kayak oar are presented. The pressure and pressure coefficient have maximum values in the central region and gradually fall toward the both sides. The CFD approach could be a useful tool to evaluate the effects of different blade designs on the oar forces and consequently on the boat propulsion contributing toward the design improvement in future oar models. The dependence of variation of pressure coefficient on the angular position of oar with respect to flow direction gives valuable information, which can be used during training for kayak competition.

\section{Method}

The closure problem of the turbulent modeling was arrived at, by using k- $\varepsilon$ model with appropriate wall functions (Bixler \& Riewald, 2002; Rouboa et al., 2006) using ANSYS FLUENT commercial CFD software. The $\mathrm{k}-\varepsilon$ model, is extensively applied and validated in the varied industrial applications (Raiesi et al., 2011). The $\mathrm{k}-\varepsilon$ model has been shown to be useful for free-shear layer flows with relatively small pressure gradients (Bardina et al., 1997). The present study is mainly focused on the variation of pressure coefficient due to angular orientation of oar blade with respect the flow direction excluding detailed flow characteristics (i.e., separation and reattachment of flow) including detailed turbulent effects. The system of equations for solving $3 \mathrm{D}$, incompressible fluid flow in steady-state regimen is as follows:

$$
\text { Continuity equation: } \frac{\partial}{\partial x_{i}}\left(\bar{U}_{i}\right)=0
$$

where $i=1,2,3$.

Navier-Stokes (momentum) equations:

$$
\begin{aligned}
& \frac{\partial}{\partial x_{j}}\left(\rho \bar{U}_{i} \bar{U}_{j}\right)= \\
& -\frac{\partial \bar{p}}{\partial x_{i}}+\frac{\partial}{\partial x_{j}}\left[\left(\mu+\mu_{t}\right)\left(\frac{\partial}{\partial x_{j}}\left(\bar{U}_{i}\right)+\frac{\partial}{\partial x_{i}}\left(\bar{U}_{j}\right)\right)-\frac{2}{3} \delta_{i j} \rho k\right]
\end{aligned}
$$

where $\bar{U}_{i}(t) \equiv \bar{U}_{i}+\bar{u}_{i}$ is the component of instantaneous velocity in $i$-direction $(\mathrm{m} / \mathrm{s}), \bar{U}_{i}$ is the component of time averaged mean velocity in $i$-direction $(\mathrm{m} / \mathrm{s}), \bar{u}_{i}$ is the component of fluctuating velocity in $i$-direction $(\mathrm{m} / \mathrm{s}), i, j$ are the direction vectors, $\rho$ is average fluid density $\left(\mathrm{kg} / \mathrm{m}^{3}\right), \mu$ is dynamic viscosity of fluid $(\mathrm{kg} / \mathrm{ms}), \mu_{\mathrm{t}}$ is turbulent viscosity of fluid $(\mathrm{kg} / \mathrm{ms}), \bar{p}$ is average pressure, $k=\frac{1}{2}\left(\bar{u}_{i} \bar{u}_{i}\right)$ the turbulent kinetic energy per unit mass $\left(\mathrm{m}^{2} / \mathrm{s}^{2}\right) ; \varepsilon$ is a dissipation rate of the turbulent kinetic energy per mass unit $\left(\mathrm{m}^{2} / \mathrm{s}^{3}\right), \delta_{i j}$ the Kronecker delta with the condition that, $\delta_{i j}=1$ if $i=j$ and $\delta_{i j}=0$ if $i \neq j$, the turbulent model constants $\sigma_{\mathrm{k}}, \sigma_{\varepsilon}, \mathrm{C}_{1}, \mathrm{C}_{2}$ are shown in Table 1 .

\section{Table 1 Modified k- $\varepsilon$ model constant}

\begin{tabular}{ccccc}
\hline $\mathbf{C}_{\boldsymbol{\mu}}$ & $\mathbf{C}_{\mathbf{1}}$ & $\mathbf{C}_{\mathbf{2}}$ & $\boldsymbol{\sigma}_{\mathbf{k}}$ & $\boldsymbol{\sigma}_{\boldsymbol{\varepsilon}}$ \\
\hline 0.09 & 1.44 & 1.92 & 1.0 & 1.3 \\
\hline
\end{tabular}


The equations of turbulence model:

$$
\begin{gathered}
\frac{\partial k}{\partial x_{i}}\left(\rho \bar{U}_{i}\right)=\frac{\partial}{\partial x_{j}}\left[\left(\mu+\frac{\mu_{t}}{\sigma_{k}}\right) \frac{\partial k}{\partial x_{j}}\right]+P_{k}-\rho \varepsilon \\
\frac{\partial \varepsilon}{\partial x_{i}}\left(\rho \bar{U}_{i}\right)=\frac{\partial}{\partial x_{j}}\left[\left(\mu+\frac{\mu_{t}}{\sigma_{\varepsilon}}\right) \frac{\partial \varepsilon}{\partial x_{j}}\right]+C_{1} P_{k} \frac{\varepsilon}{k}-C_{2} \rho \frac{\varepsilon^{2}}{k}
\end{gathered}
$$

The production of kinetic energy term is given as:

$$
P_{k}=-\rho \bar{u}_{i} u_{j} \frac{\partial \bar{U}_{j}}{\partial x_{i}}=\mu_{t} S^{2}
$$

where $S$ the modulus of the mean rate-of-strain tensor is given by

$$
S \equiv \sqrt{2 S_{i j} S_{i j}}
$$

where the mean strain rate $S_{i j}$ is defined by

$$
S_{i j}=\frac{1}{2}\left[\frac{\partial U_{i}}{\partial x_{j}}+\frac{\partial U_{j}}{\partial x_{i}}\right]
$$

The turbulent viscosity is calculated by the following relation:

$$
\mu_{t}=\rho C_{\mu} \frac{k^{2}}{\varepsilon}
$$

To limit numerical dissipation, particularly when the geometry is complex inducing an unstructured grid, secondorder discretization schemes are used (Figure 1). In generic terms, the convergence of the calculation is checked by the value of the residuals of the various flow parameters. The convergence criteria in ANSYS FLUENT was set at $10^{-6}$. This criterion is assumed sufficient to ensure the convergence of the solution for the current study. The boundary layer was created with aspect ratio algorithm with 4 rows of boundary layer grid cells with transition pattern of 1:1, with first row of grid at $0.01 \mathrm{~mm}$ immediately preceding wall grid with last percentage of 50\% maintaining internal continuity. The first cell was $0.01 \mathrm{~mm}$ away from the blade producing mean $\mathrm{y}^{+}$value was 1.09 and the tetrahedral grids had maximum skewness of 0.74 and overall average skewness of 0.38. Appropriate number of tetrahedral grids cells in simulation model was arrived, which was an outcome of grid independence test carried out at the beginning of actual simulations. It was found that the difference in solutions for the drag coefficients for subsequent refinement in tetrahedral grid were less than $1 \%$, when tested at an angle of $90^{\circ}$ to flow direction.

\section{Oar Blade Geometric Model}

For this first study on CFD applied on oar motion in water, the most common blade was chosen (Macon model), which presented symmetrical form in relation to the oar axis (Figure 2).

The blade used in the numeric simulation was first modeled using a CAD commercial Software (Solidworks Inc, Concord, USA) to compute the 3D geometrical model (Figure 3). The oar blade front face surface area is $0.12758742 \mathrm{~m}^{2}$, length of blade is $0.72 \mathrm{~m}$, width is $0.2 \mathrm{~m}$.

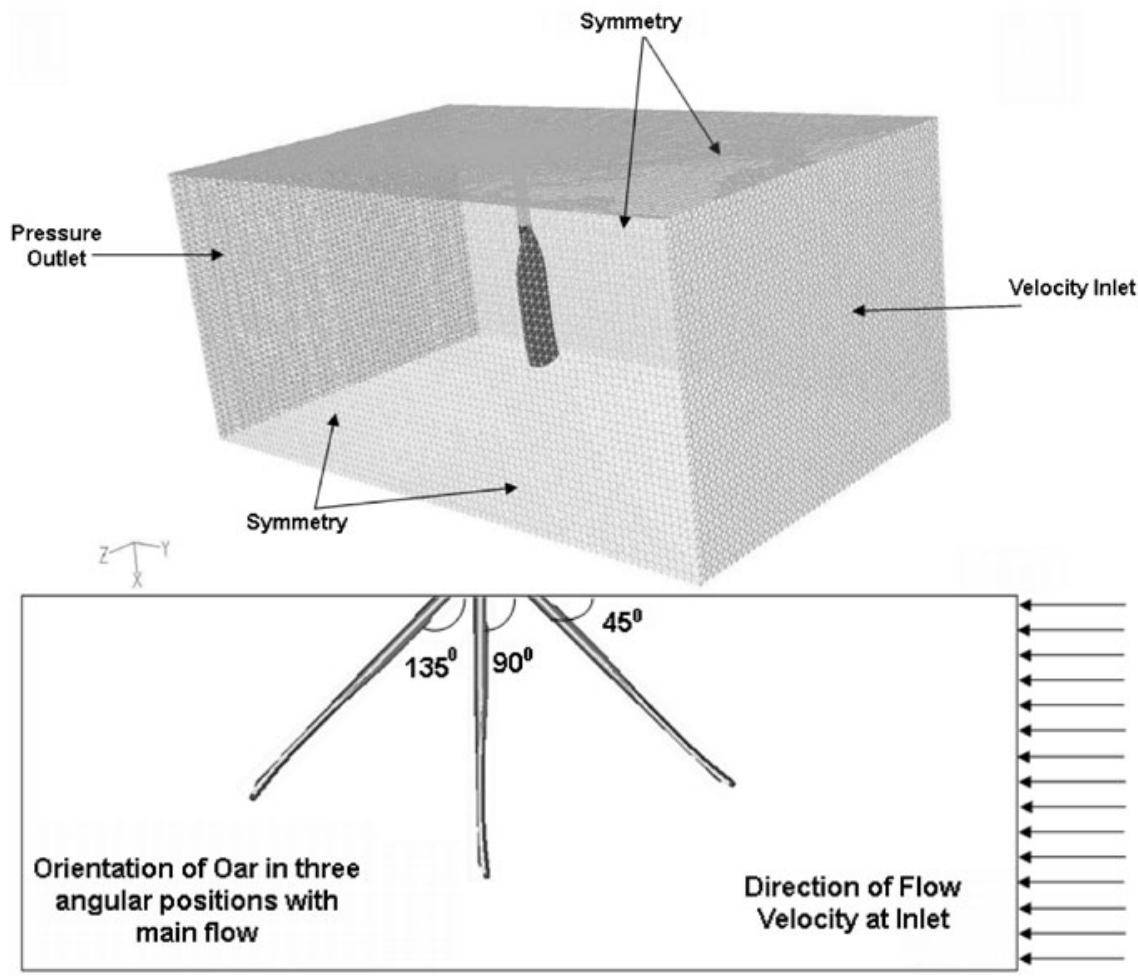

Figure 1 - The computational mesh model showing boundary condition along with orientation of oar blade for individual case under study. 


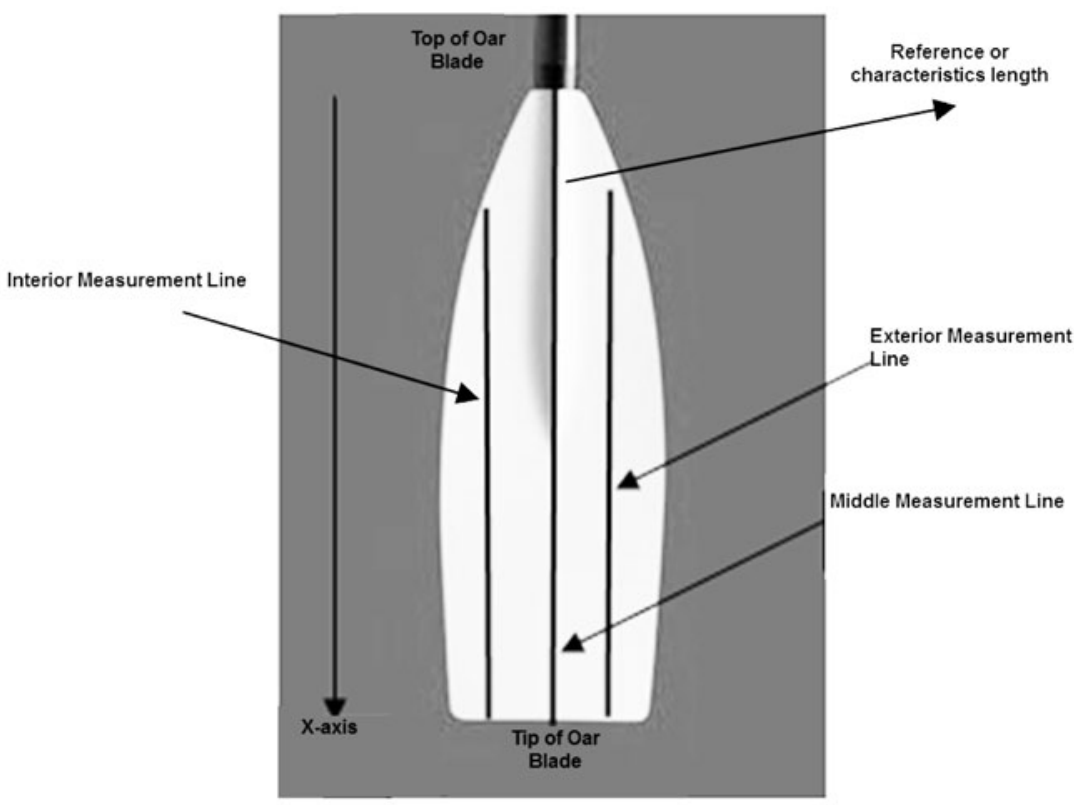

Figure 2 - The blade reference model showing location of lines studied.

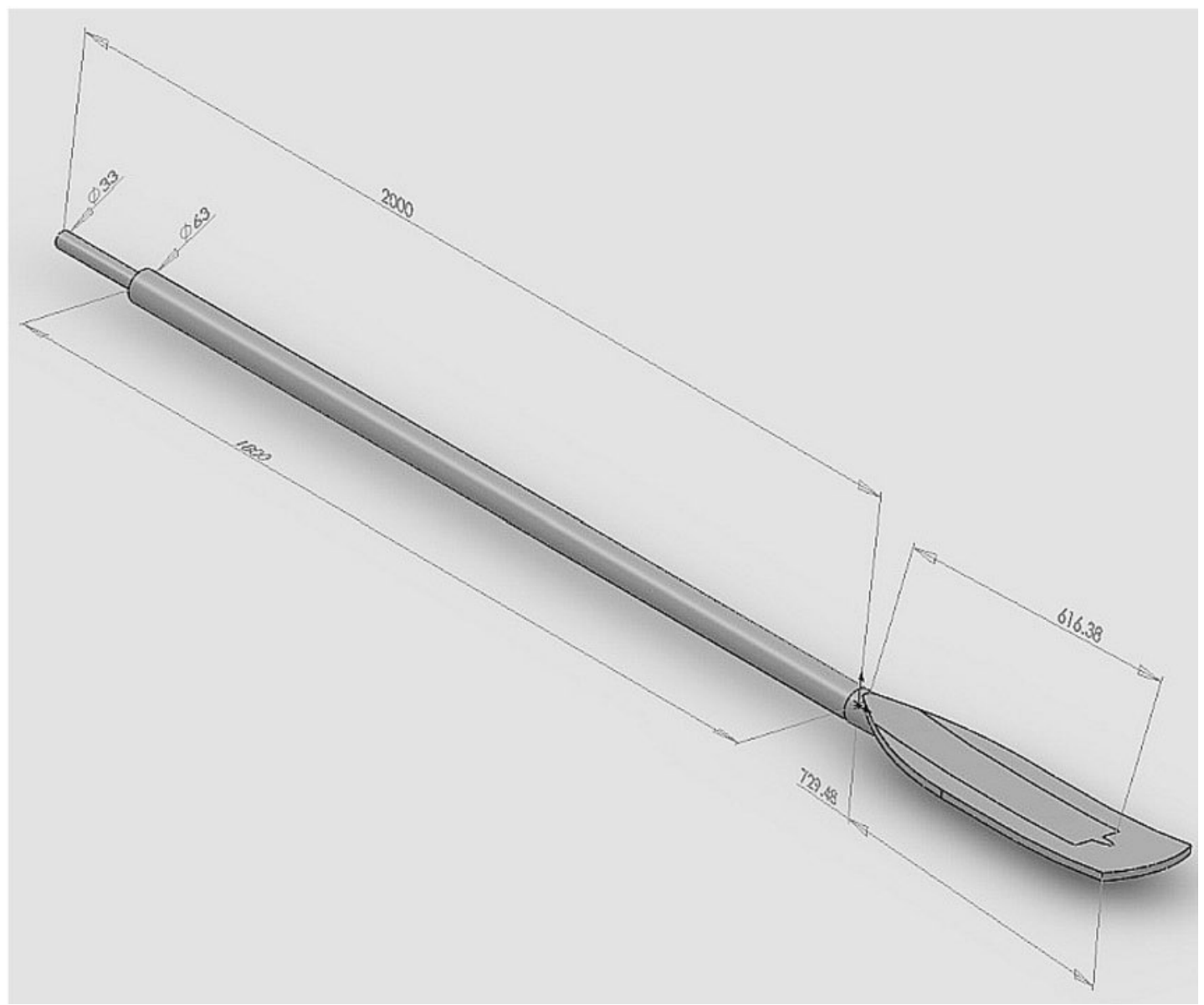

Figure 3 - Computational geometrical model from SOLIDWORKS software. 
The 3D model is positioned in a rectangular geometrical domain and is meshed in GAMBIT (ANSYS FLUENT Inc, Sacramento, California) commercial meshing software. The boundary conditions were applied, the wall boundary condition of the oar surface, inlet and outlet surface at side surfaces and the symmetric boundary condition on the remaining exterior surfaces. Finally, the mesh model was exported to FLUENT software to simulate the flows around the oar.

The flow conditions were considered as following:

- for this first approach of CFD in kayak, the flow was considered as steady state with a horizontal velocity, $\mathrm{U}$ of $4 \mathrm{~m} / \mathrm{s}$, which corresponds to a Reynolds number $(\operatorname{Re}=\mathrm{UL} / \mathrm{v}$, with $\mathrm{L}$ denoting the blade's length of $0.72 \mathrm{~m}$ and $v$ the fluid kinematic viscosity of water). As an initial condition, the water was assumed to be at rest.

- The pressure was set equal to zero for all the sides of the computational domain.

- The fluid was considered incompressible with density of $\rho=996.6 \mathrm{~kg} \cdot \mathrm{m}^{-3}$ and dynamic viscosity of water is $8.90 \times 10^{-4} \mathrm{~Pa} \cdot \mathrm{s}$ at $25^{\circ} \mathrm{C}$.

- The action of the gravity force was set at $\mathrm{g}=9.81$ $\mathrm{m} \cdot \mathrm{s}^{-2}$ with the assumption of turbulence intensity of $1 \%$ at the inlet.

The oar was positioned stationery in the central part of the domain, the water flowed perpendicular to the oar. To simulate the different phases of the stroke, the flow was modeled for three different positions of the oar: $45^{\circ}$, which corresponded to the catch phase of the water; $90^{\circ}$, the major propulsive phase, which corresponds to the compressive phase (power phase); and $135^{\circ}$, which corresponds to the end of the aquatic phase (Begon, 2006). The evaluation of the distribution of the local pressure on the oar was carried out along three imaginary lines along the length of oar blade, each on the front and rear sides of the oar blade. The location of these three lines were taken judiciously so as to reflect the average of pressure variation in nearby of three region i.e., internal, middle, external respectively (Figure 2) as per the location of the individual lines, which entailed the detailed analysis of pressure variation. This analysis can contribute in more specific geometric design modifications to decrease the drag by minimization of drag contributing regions of surface area respectively. The FLUENT simulation results allowed to get the $\mathrm{C}_{\mathrm{p}}$ for each $\mathrm{x}$-coordinate of each line (internal, middle, external), for each side (front versus rear) and for each oar angle $\left(45^{\circ}, 90^{\circ}, 135^{\circ}\right)$. The study of variation of $\mathrm{C}_{\mathrm{p}}$ along the respective lines was carried out as $\mathrm{C}_{\mathrm{p}}$ was most important component contributing toward total drag coefficient and the propulsion obtained from the oar emerges to be essentially based on this drag component. The drag coefficient is obtained by the following Equations (9):

$$
C_{D}=\frac{F_{\text {drag }}}{1 / 2 \rho \cdot V^{2} \cdot A_{\text {projected }}}
$$

where $F_{\text {drag }}$ is drag force (about $2143 \mathrm{~N}$ ), $\mathrm{C}_{\mathrm{D}}$ is drag coefficient, $\rho$ the average fluid density, $\mathrm{V}$ is average fluid velocity, $A_{\text {projected }}$ is the average projected cross-section area (about $0.1333508 \mathrm{~m}^{2}$ ) in the flow direction, all in S.I. units. The $t$ test was applied to test for the difference in means for the different lines, the different sides, and the different angles $(p<.05)$ (Ockerman \& Goldsman, 1999).

\section{Results}

Results indicated that irrespective of the oar angle, the three lines of the front side of the oar presented the similar shape of $C_{p}$ curve along the $x$-axis (Figure 4). For the three different orientations $\left(45^{\circ}, 90^{\circ}\right.$, and $\left.135^{\circ}\right)$ the $\mathrm{C}_{\mathrm{p}}$ curve could be divided into three parts. The first part between 0 and $0.3 \mathrm{x}$, which corresponded to the upper part of the blade (between the end of the handle and the beginning of the blade). For this first part, $\mathrm{C}_{\mathrm{p}}$ is different for the three different lines with a decrease for the middle line, an increase and then a decrease for the external and internal lines of drag coefficient. A constant drag coefficient characterized the second portion of the curve between 0.3 at $0.5 \mathrm{x}$ for the three lines (the middle part of the blade). The third part of the curve located between $0.5-0.8 \mathrm{x}$ (the end of the blade) presented similar $\mathrm{C}_{\mathrm{p}}$ for the three lines with an increase from 0.5 to $0.7 \mathrm{x}$ and decreases from 0.7 to $0.8 \mathrm{x}$. Thus for all lines and all angles $\left(45^{\circ}, 90^{\circ}, 135^{\circ}\right)$, superior and unsteady $C_{p}$ values were observed at the extremities of the blade, with stable lower $\mathrm{C}_{\mathrm{p}}$ for the central part of the blade. These results clearly indicated a non-uniformity of $\mathrm{C}_{\mathrm{p}}$ on the front face of the paddle especially at the extremities of the oar.

Although there is similarity of the $C_{p}$ curves observed for the three oar angles, different values were observed for different angles of orientation with the main flow. The comparison of the stable portion showed greater $\mathrm{C}_{\mathrm{p}}$ for an oar angle of $90^{\circ}$ compared with $45^{\circ}$ and $135^{\circ}$ angles. To evaluate these differences, we compared the $\mathrm{C}_{\mathrm{p}}$ of the three lines during the steady portion of the curve $(0.3-0.5 \mathrm{x})$ for the front side. Results of the $t$ test indicated significant differences between the $C_{p}$ at $45^{\circ}$ and $90^{\circ}(p=.00532), 90^{\circ}$ and $135^{\circ}(p=.0012)$ and not between $45^{\circ}$ and $135^{\circ}(p=.875)$. In other words, the oar force is greater, when the oar is perpendicular to the boat with no significant differences among the three lines indicating an homogeneous distribution of $\mathrm{C}_{\mathrm{p}}$ values, on the blade (Figure $5 \mathrm{~b}$ ). For the $45^{\circ}$ blade angle, the $\mathrm{C}_{\mathrm{p}}$ was higher for the external line of the blade than for the middle and interior ones (Figure 5a). Opposite results were observed for the $135^{\circ}$ blade angle with higher $C_{p}$ for the interior line than for the middle and exterior lines (Figure 5c).

The front side, presented minor variation of $\mathrm{C}_{\mathrm{p}}$ values whatever the orientation of the oar, and whatever the line of the oar (Figure 6). $C_{p}$ is present on the entire front side of the blade when the pressure appeared to vary only on few areas of the rear side with differences from one line to another. We can also observe that for both sides, the 

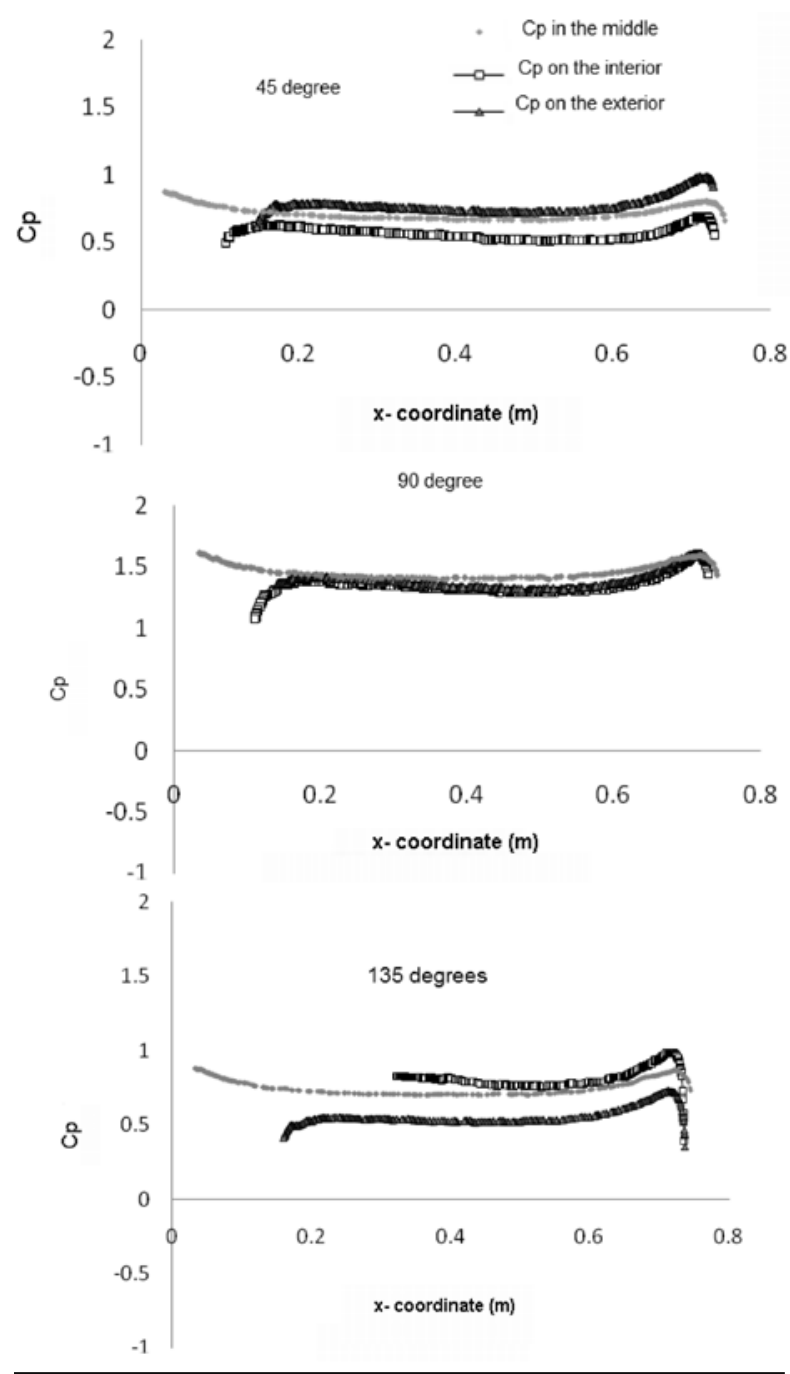

Figure $4-$ Pressure coefficients $\left(\mathrm{C}_{\mathrm{p}}\right)$ on the $\mathrm{x}$-coordinates for the three angles $\left(45^{\circ}, 90^{\circ}, 135^{\circ}\right)$ for the three lines (interior, middle, exterior) of the front side.

pressure on areas appeared to vary similar whatever the orientation of the blade.

Greater $C_{p}$ was observed on the front face of the blade than on the rear one (Figure 7), whatever the orientation of the blade was $\left(45^{\circ}, 90^{\circ}\right.$ and $\left.135^{\circ}\right)$.

The pathlines plot colored with average flow velocity starting from frontal face of oar blade showing recirculation zone at rear face of oar blade and contour plots of average velocity around the kayak oar with rear side view, the pressure and pressure coefficient on the front side of kayak oar are presented in the Figure 8. The pressure and pressure coefficient have maximum values in the central region and gradually fall toward the both sides.

\section{Discussion}

For the first CFD study on kayak, the main goal was to evaluate the influence of blade angle on the flow. We hypothesized that (i) the pressure and the local pressure coefficient were not uniform regarding the surface of the

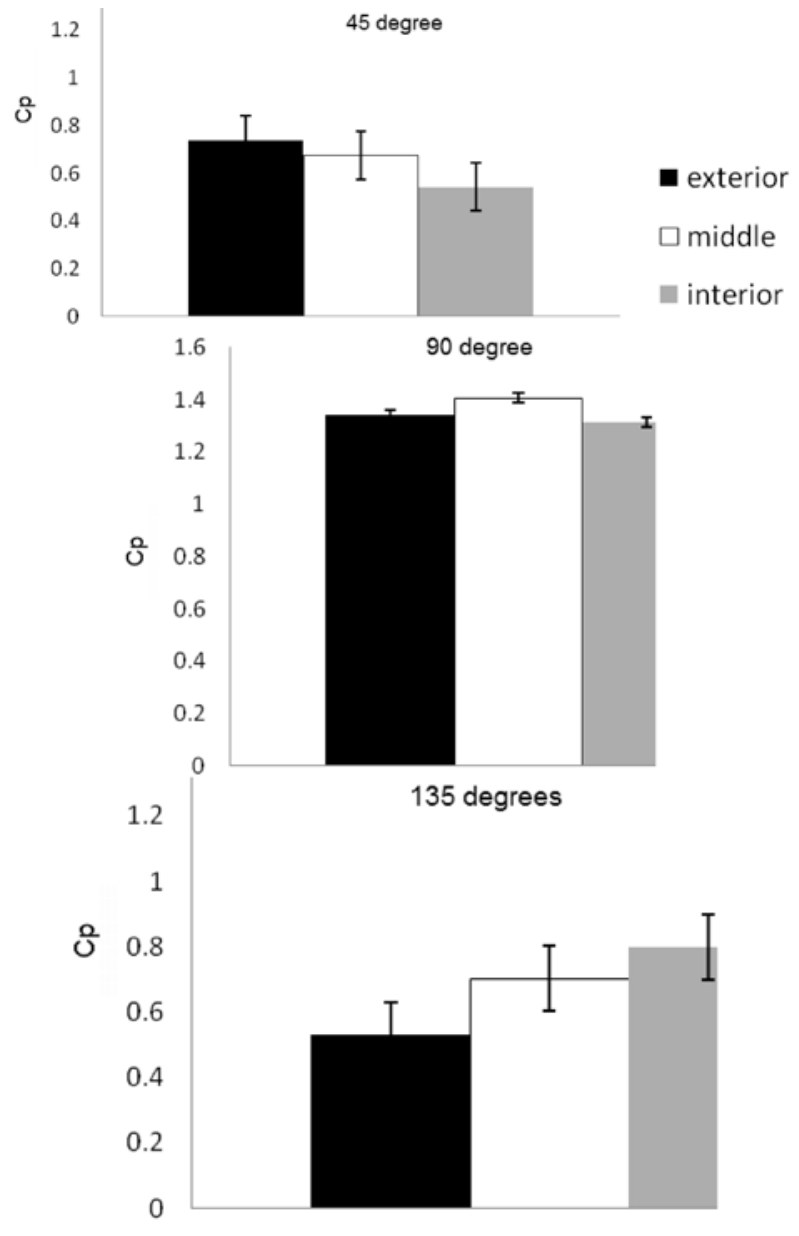

Figure 5 - Evolution of $\mathrm{C}_{\mathrm{p}}$ for three attack angles $\left(45^{\circ}, 90^{\circ}\right.$, $135^{\circ}$ ) for the three lines of the front side (interior, middle, exterior) at $4 \mathrm{~m} / \mathrm{s}$.
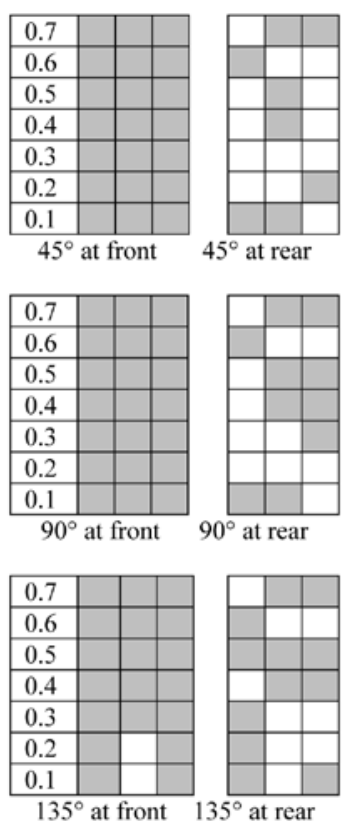

Figure 6 - Presence of the $\mathrm{C}_{\mathrm{p}}$ (gray) and $\mathrm{C}_{\mathrm{p}}$ null (white) for the three lines, for both sides (front versus rear) and for three orientations of the oar. 


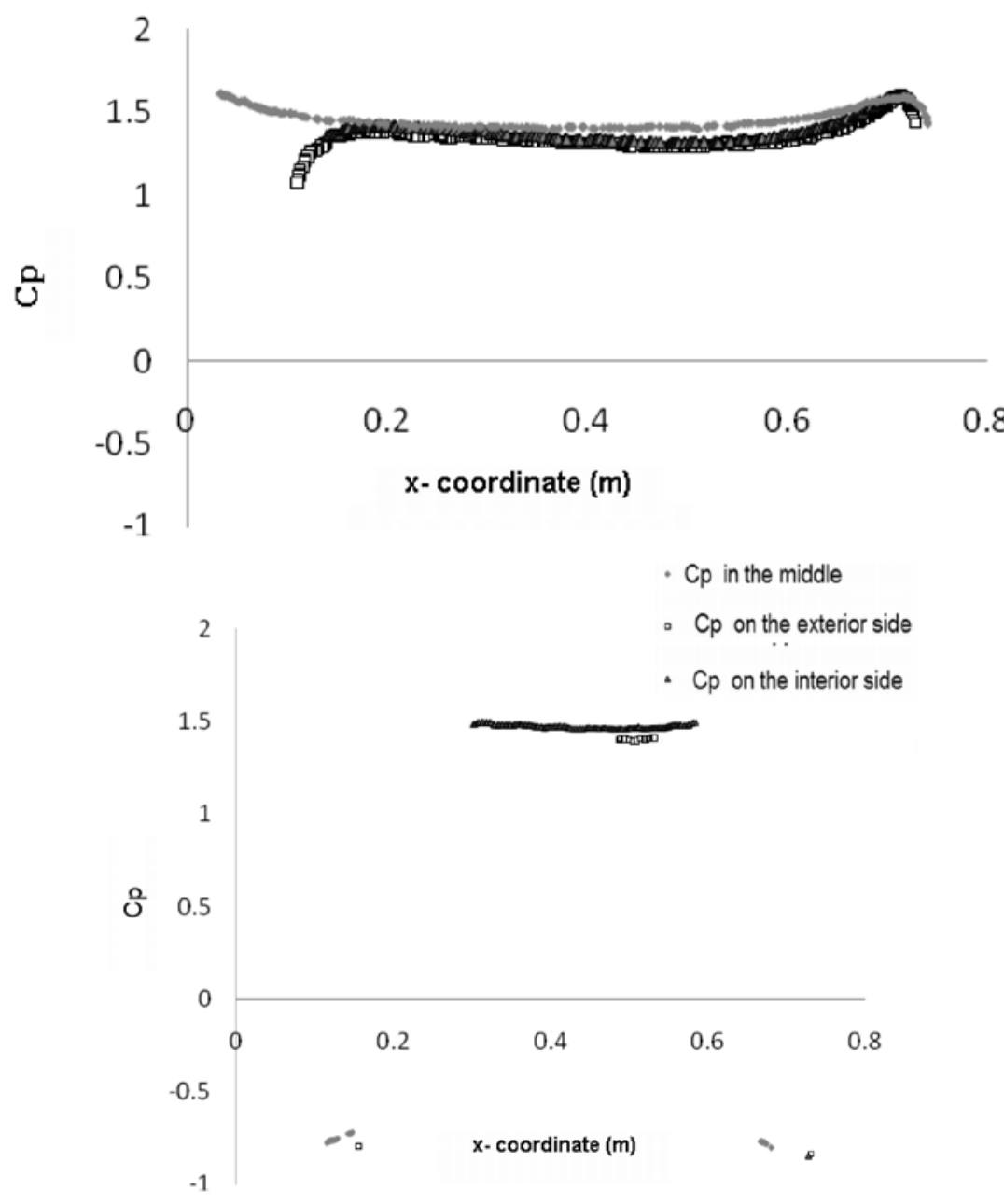

Figure 7 - Pressure coefficients $\left(\mathrm{C}_{\mathrm{p}}\right)$ on the $\mathrm{x}$-coordinates for the three lines (interior, middle, exterior) of the front and rear sides at $90^{\circ}$.

blade (internal, middle and external portions) and the side of the blade (front and rear), and (ii) the local pressure coefficient changed with the orientation of the blade with a maximum at $90^{\circ}$ orientation angle of the blade.

Results pointed out that even if the fluid flow exerts different pressures on the blade, similar patterns of the local pressure coefficient on the $\mathrm{x}$-axis were observed for the three blade angles $\left(45^{\circ}, 90^{\circ}, 135^{\circ}\right)$ and for the three lines (internal, middle, external) of the blade with higher values at the extremities and lower stable values for the central portion for the front side. As observed for the fin fish (Lauder et al., 2007; Zhu et al., 2008), the pressure is not uniform on all the surface of the blade reflecting different flow motions. The greater $\mathrm{C}_{\mathrm{p}}$ at the handle-blade junction is like the greater $\mathrm{C}_{\mathrm{p}}$ observed at the fin-body junction of the fish (Zhu et al., 2008). At this junction, water could not circulate around the handle-blade system (or body-fin for the fish) and, consequently, the pressure increased. The greater $C_{p}$ at the free extremity could be due to the smaller rounded surface at the end of the blade. A gradual increase of the local pressure coefficient was observed (Zhang et al., 2006) from the fin base to the fin tip similar to the observed $C_{p}$ increase from the top to the tip of the blade in present study. The stability and the lower $\mathrm{C}_{\mathrm{p}}$ in the central portion of the blade could be due to the greater area and the uniform design of this portion.

Despite the same $C_{p}$ patterns for the three lines and the three angles, results showed higher values for the blade angle of $90^{\circ}$ and lower ones for $45^{\circ}$ and $135^{\circ}$. The fluid did not exert the same force at $45^{\circ}, 90^{\circ}$ and $135^{\circ}$. These results were similar to those observed in swimming, with higher $C_{p}$ for an attack hand angle of $90^{\circ}$ either quantified from a kinematic approach (Schleihauf, 1974) or from CFD (Bixler \& Riewald, 2002; Rouboa et al., 2006; Marinho et al., 2010). In addition, for $90^{\circ} \mathrm{C}_{\mathrm{p}}$ is not significantly different for all the portions of the blade (internal, middle and external). For this angle, the blade is perpendicular to the fluid flow, so the fluid hits this zone uniformly. In this position, the fluid cannot go around the blade, and consequently no vortex and no lift force can be created. In this case, the only propulsive force is the drag force applied uniformly on all the surface of the blade.

The nonsignificant differences between $\mathrm{C}_{\mathrm{p}}$ values for $45^{\circ}$ and $135^{\circ}$ indicated that the fluid globally exerted the 


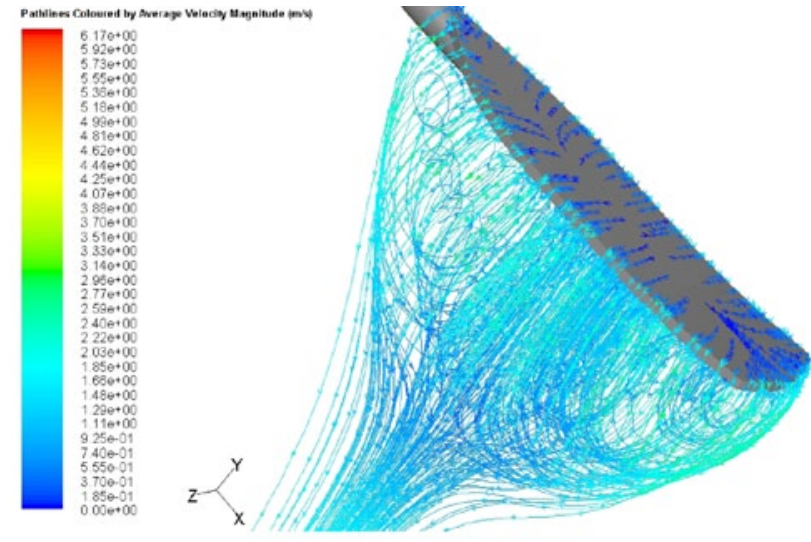

(a)

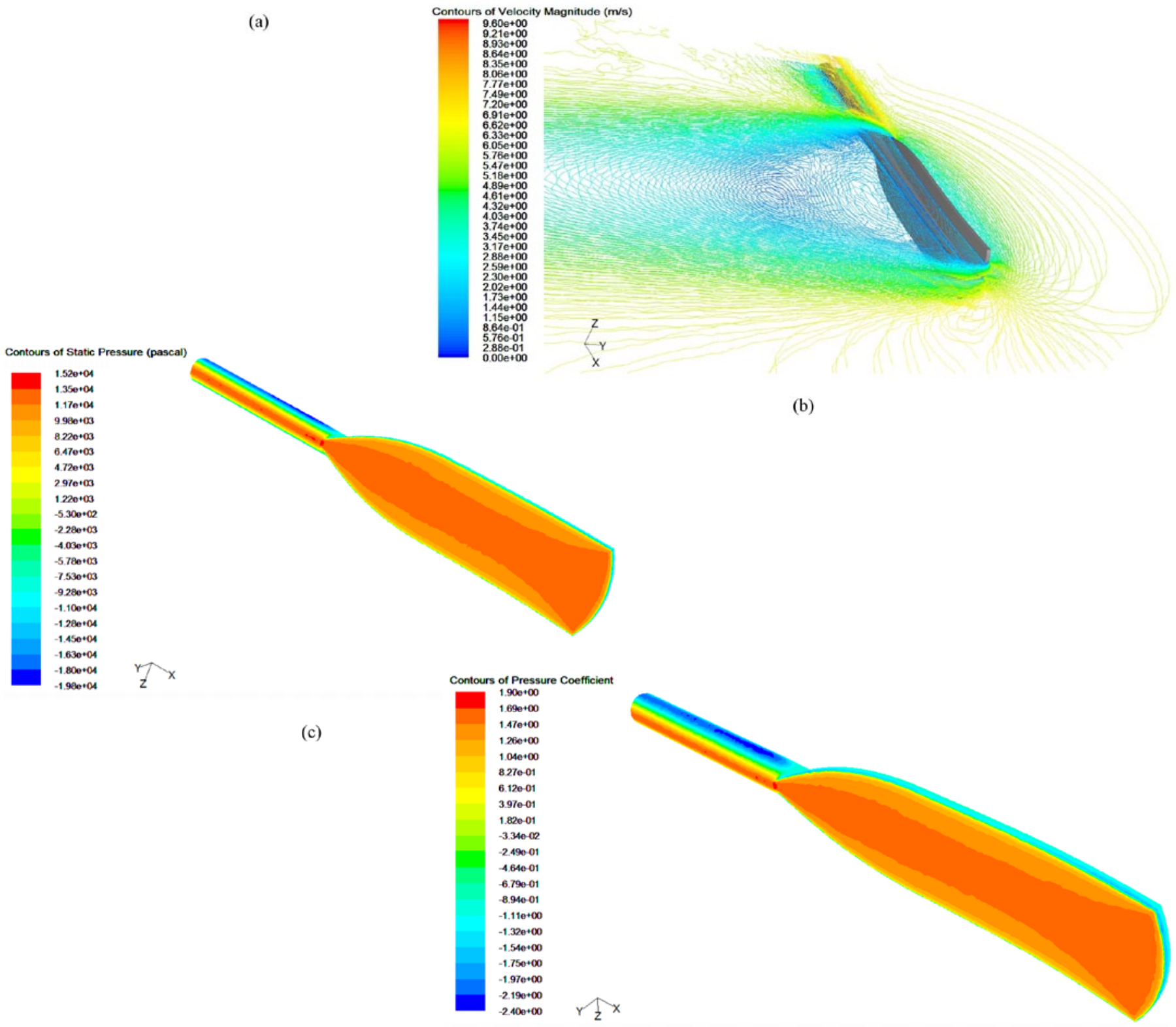

(d)

Figure 8 - For velocity of $4 \mathrm{~m} / \mathrm{s}$ and 90 degree angle of oar with respect to the flow direction the (a) pathlines colored with average velocity starting from frontal face of oar blade, (a) contours of average velocity around the kayak oar blade, (b) contours of average pressure on kayak oar blade, (c) contours of average pressure coefficient of the kayak oar blade. (The online PDF version of this article contains this figure in color.) 
same pressure at the beginning and at the end of the oar motion in the water. Despite the similarities of $\mathrm{C}_{\mathrm{p}}$ values, the distribution of the $\mathrm{C}_{\mathrm{p}}$ is not the same for $45^{\circ}$ and $135^{\circ}$, with superior $\mathrm{C}_{\mathrm{p}}$ for the external edge compared with the internal one for $45^{\circ}$, and converse by for $135^{\circ}$. These differences between the internal and external edges could reflect differences in lift forces for these angles and/or differences in vortex productions. Similar results have been observed on the fish locomotion (Liao, 2007). For example, a counter-clockwise vortex is visible at the head when the fish-body presented an angle of $100^{\circ}$ with the direction of the fluid. The vortex moved to the middle of the body when the fish was at $180^{\circ}$, i.e., in the same direction as the fluid flow. It was concluded (Zhang et al., 2006) that the pressure is quite small at the leading edge area to reach its maximum at the trailing edge area. Thus, the repartition of $\mathrm{C}_{\mathrm{p}}$ values changed with the orientation of the oar, as did the undulation of the fish.

Finally big differences in $C_{p}$ values were observed between the rear and front sides of the oar. For the three blade angles, negative $C_{p}$ was observed for the rear side and positive $\mathrm{C}_{\mathrm{p}}$ for the front one. Moreover, the rear side presented some areas without any noticeable drag contributions. Similar results were observed for the swimmer, for which the boundary layer separation resulted in formation of a low-pressure region behind the body (Naemi et al., 2009) and also on the fish. It was noticed (Dubois et al., 1974) that the pressure was positive in front of the fish and became negative at the back portion. It was concluded (Hirata, 2001) that pressure is negative at the opposite side of the propulsive side when the fish is swimming. The negative pressure can be explained from the Bernoulli principle: the water accelerated to pass around the fish, resulting in a decrease of the pressure (Dubois et al., 1974). The pressure difference between the front and rear sides created a force oriented from the high to the low pressures areas, i.e., from the front to the rear sides contributing to propel the boat.

The present study represented the application of CFD approach of the flow around the oar in kayaking. Results indicated that the angle of the oar strongly influenced the $C_{p}$ with maximum values for a $90^{\circ}$ angle of the oar. Moreover, the distribution of the pressures is different for the internal and external edges depending of the oar angle. Finally, the difference of negative $\mathrm{C}_{\mathrm{p}}$ in the rear side and the positive ones in the front side contributed to create a propulsive force. The CFD approach could be a useful tool to evaluate the effects of different blade designs on the oar forces and consequently on the boat propulsion contributing toward development of new oar design.

\section{References}

Ackland, T.R., Ong, K., Kerr, D.A., \& Ridge, B. (2003). Morphological characteristics of Olympic sprint canoe and kayak paddlers. Journal of Science and Medicine in Sport, 6, 285-294. PubMed doi:10.1016/S14402440(03)80022-1

Aitken, D.A., \& Neal, R.J. (1992). An On-Water Analysis System for Quantifying Stroke Force Characteristics during Kayak Events. International Journal of Sport Biomechanics, 8, 165-173.

Alves, F., Marinho, D., Leal, L., Rouboa, A., \& Silva, A. (2007). 3-D computational fluid dynamics of the hand and forearm in swimming. Medicine and Science in Sports and Exercise, 9, 339.

Bardina, J.E., Huang, P.G., \& Coakley, T.J. (1997). Turbulence Modeling Validation, Testing, and Development. NASA Technical Report Memorandum, 110446. Retrieved from http://www.ewp.rpi.edu/hartford/ jon gel/ /19970017828_1997026179.pdf

Bégon, M. (2006). Analysis and three-dimensional simulation of cyclical movements in a specific kayak ergometer (Unpublished doctoral dissertation, in French). University of Poitier, Graduate School of Science Engineering, France.

Berger, M.A.M., de Groot, G., \& Hollander, A.P. (1995). Hydrodynamic drag and lift forces on human hand/arm models. Journal of Biomechanics, 28, 125-133. PubMed doi:10.1016/0021-9290(94)00053-7

Berger, M.A.M., de Groot, G., \& Hollander, A.P. (1997). Influence of hand shape on force generation during swimming. In B.O. Eriksson, \& L. Gullstrand (Eds.), Proceedings of XII FINA World Congress on Sports Medicine. (pp. 389-396). Goteborg: Chalmers University Reproservice. ISBN 91-630-6228-3.

Bixler, B.S., \& Riewald, S. (2002). Analysis of swimmer's hand and arm in steady flow conditions using computational fluid dynamics. Journal of Biomechanics, 35, 713-717. PubMed doi:10.1016/S0021-9290(01)00246-9

Di Puccio, F., \& Mattei, L. (2008). Kayak rowing: Kinematic simulation of different techniques. Journal of Biomechanics, 41, 425-433. doi:10.1016/S0021-9290(08)70424-X

Dolnik, A., \& Krasnopievtsev, I. (1980). Les fréquences de pagayage en canoe-kayak-USSR-Trad. Grebnoj Sport, 589, 39-43 (In Russian).

Dubois, A.B., Cavagna, G.A., \& Fox, R.S. (1974). Pressure distribution on the body surface of swimming fish. The Journal of Experimental Biology, 60, 581-591. PubMed

Gardano, P., \& Dabnichki, P. (2005). On hydrodynamics of drag and lift of the human arm. Journal of Biomechanics, 39, 2767-2773. PubMed doi:10.1016/j.jbiomech.2005.10.005

Gourgoulis, V., Aggeloussis, N., Vezos, N., Antoniou, P., \& Mavromatis, G. (2008). Estimation of hand forces and propelling efficiency during front crawl swimming with hand paddles. Journal of Biomechanics, 41, 208-215. PubMed doi:10.1016/j.jbiomech.2007.06.032

Grare, A. (1985). Study and realization of a data processing system for a flatwater kayak. (Unpublished doctoral dissertation, in French). University of Science and Technology of Lille, France.

Hirata, K. (2001). Principles of the Swimming Fish Robot (Unpublished master thesis). National Maritime Research Institute, Japan. Introduction available at http://www.nmri. go.jp/eng/khirata/fish/general/principle/index_e.html

Issurin, V.B. (1980). The mechanism of stroke. Theory and Practice in Physical Culture, 6, 50-53.

Issurin, V.B., Kransnov, E.A., \& Ramuzov, G.G. (1983). Comparative effectiveness of different variants of the technique of paddling in canoe-kayak. Theory and Practice in Physical Culture, 9, 147-152.

Kendal, S.J., \& Sanders, R.H. (1992). The technique of elite flatwater kayak paddlers using the wing paddle. International Journal of Sports Biomechanics, 8, 233-250.

Lauder, G.V., Anderson, E.J., Tangorra, J., \& Madden, P.G.A. (2007). Fish biorobotics: Kinematics and hydrodynamics 
of self propulsion. The Journal of Experimental Biology, 210, 2767-2780. PubMed doi:10.1242/jeb.000265

Liao, J.C. (2007). A review of fish swimming mechanics and behaviour in altered flows. Philosophical Transactions of the Royal Society B. Biological Sciences, 362, 1973-1993. PubMed doi:10.1098/rstb.2007.2082

Mann, R.V., \& Kearney, J.T. (1980). A biomechanical analysis of the Olympic-style flatwater kayak stroke. Medicine and Science in Sports and Exercise, 12, 183-188. PubMed doi:10.1249/00005768-198023000-00010

Marinho, D.A. (2008). The study of swimming propulsion using computational fluid dynamics. A three dimensional analysis of the swimmer's hand and forearm (Unpublished doctoral dissertation). Universidade de Tràs-os-Montes E alto Douro, Vila Real, Portugal.

Marinho, D.A., Barbosa, T.M., Reis, V.M., Kjendlie, P.L., Alves, F.B., Vilas-Boas, J.P., . . . Rouboa, A.I. (2010). Swimming propulsion forces are enhanced by a small finger spread. Journal of Applied Biomechanics, 26, 87-92. PubMed

Minetti, A.E., Machtsiras, G., \& Masters, J.C. (2009). The Optimum Finger Spacing In Human Swimming. Journal of Biomechanics, 42, 2188-2190. PubMed doi:10.1016/j. jbiomech.2009.06.012

Naemi, R., Chockalingam, N., Dunning, D.N., \& Chevalier, T.L. (2009). A new method of measuring the Hydrodynamic Glide Efficiency in Swimming. A review. Journal of Science and Medicine in Sport, 13, 444-448. PubMed doi:10.1016/j.jsams.2009.04.009

Novakova, H., \& Sukopp, J. (1979). Kinematic analysis of the art of canoe paddling speed. Theory of Physical Education, 27, 234-238 (In Czech).

Ockerman, D.H., \& Goldsman, D. (1999). Student t-tests and compound tests to detect transients in simulated time series. European Journal of Operational Research, 116, 681-691. doi:10.1016/S0377-2217(98)00233-1

Plagenhoef, S. (1979). Biomechanical Analysis Of Olympic Flatwater Kayaking and Canoeing. Research Quarterly, 50, 443-459. PubMed

Raiesi, H., Piomelli, U., \& Pollard, A. (2011). Evaluation of Turbulence Models Using Direct Numerical and Large-
Eddy Simulation Data. Journal of Fluids Engineering, 133, 193-203. doi:10.1115/1.4003425

Rouboa, A., Silva, A., Leal, L., Rocha, J., \& Alves, F. (2006). The effect of swimmer's hand/forearm acceleration on propulsive forces generation using Computational Fluid Dynamics. Journal of Biomechanics, 39, 1239-1248. PubMed doi:10.1016/j.jbiomech.2005.03.012

Rushall, B.S., Sprigings, E.J., Holt, L.E., \& Cappaert, J.M. (1994). A re-evaluation of forces in swimming. Journal of Swimming Research, 10, 6-30.

Schleihauf, R.E. (1974). A biomechanical analysis of freestyle. Swimming Technique, 11, 89-96.

Silva, A., Rouboa, A., Leal, L., Rocha, J., Alves, F., Moreira, A., . . . Vilas-Boas, J.P. (2005). Measurement of swimmer's hand/forearm propulsive forces generation using computational fluid dynamics. Portuguese Journal of Sport Sciences, 5, 288-297.

Takagi, H., Shimizu, Y., Kurahima, A., \& Sanders, R.H. (2001). Effect of thumb abduction and adduction on hydrodynamic characteristics of a model of the human hand. In J. Blackwell and R.H. Sanders (Eds.) Proceedings of Swim Sessions XIX International Symposium on Biomechanics in Sports (pp. 122-126). San Francisco, California, June 26, 2001.

Wargnier, J.B. (1990). Studies in situ different Kayak Flatwater (Unpublished Master's Project, in French). Free Institute Superior of EP, France.

Zaidi, H., Taiar, R., Fohano, S., \& Polidori, G. (2008). Analysis of the effect of swimmer's head position swimming performance using computational fluid dynamics. Journal of Biomechanics, 41, 1350-1358. PubMed doi:10.1016/j. jbiomech.2008.02.005

Zhang, Y.H., He, J.H., Yang, J., \& Zhang, A.W. (2006). A Computational Fluid Dynamics (CFD) Analysis of an Undulatory Mechanical Fin Driven by Shape Memory Alloy. International. Journal of Automation and Computing, 4, 374-381. doi:10.1007/s11633-006-0374-4

Zhu, Q., \& Shoele, K. (2008). Propulsion performance of a skeleton strengthened fin. The Journal of Experimental Biology, 211, 2087-2100. PubMed doi:10.1242/jeb. 016279 